\title{
Correlative Spectromicroscopy and Tomography Involving Soft X-ray Methods
}

\author{
Adam P. Hitchcock ${ }^{1}$, Xiaoyue Wang ${ }^{2}$ and Kathryn Grandfield ${ }^{2}$ \\ 1. Chemistry and Chemical Biology, McMaster University, Hamilton, Canada \\ 2. Materials Science and Engineering, McMaster University, Hamilton, Canada
}

Many important scientific and technical problems are best addressed using multiple analytical techniques which combine the strengths of complementary methods. Here we provide examples from bio-material interfaces, biomineralization and material science which combine synchrotron-based scanning transmission X-ray microscopy (STXM) with transmission electron microscopy ((S)TEM), electron tomography (ET), EELS tomography, atom probe tomography (APT). atomic force microscopy (AFM) and infrared microscopy (FTIR). STXM provides X-ray absorption based chemical sensitivity at mesoscale resolution $(10-30 \mathrm{~nm})$ which complements the higher spatial resolution EM, APT and AFM techniques, and the lower resolution, but more chemically sensitive FTIR. Figure 1 outlines the approximate position in spatial resolution - chemical sensitivity 'space' of the methods used.

The interface between human bone and artificial implants is critical for osseointegration, the stimulation of mineralized bone which forms a long-lasting load-bearing bond [1]. We have used chemically sensitive correlative imaging by X-ray tomography (CT), TEM, ET, STEM, APT and STXM to investigate the 4D (3D chemical) structure of the interface of human bone and a Ti dental implant, that had been in place for 47 months [2]. Figure 2 presents results from ET, EELS tomography and APT of a FIB sample needle. The ability to perform $3 d$ measurements at several length scales with chemical sensitivity provides insights not available from any single method. The bone near the interface has been remodeled. The Z-contrast from on-axis HAADF-STEM ET (Fig. 2a) differentiates Ti implant, collagen and apatite. There is evidence of continuous bone growth and integration with the nano-topographic oxide layer on the surface of the laser-modified, commercially pure, titanium dental implant. The reconstruction of the EELS tomography on the needle sharpened to $100 \mathrm{~nm}(\mathbf{F i g}$. 2b) provides higher resolution elemental mapping of the interface, specifically showing the $\mathrm{Ca}$ of the bone apatite (green), and organic components (red). The needle was then FIB sharpened to $50 \mathrm{~nm}$, and subjected to APT. Fig. 2c shows that both $\mathrm{Ca}$ and $\mathrm{C}$ are in contact with the outer Ti oxide layer of the implant, and reveals a buried Ti nitride layer in the implant. This correlative tomography workflow helps to visualize the inhomogeneous and hierarchical bone-implant interface, and it is also applicable to the investigation of other complex biointerphases.

More detailed chemical speciation was provided by 2D TEM-EELS at the Ca $2 p$ edge, as well as STXM studies at the $\mathrm{C} 1 \mathrm{~s}$, $\mathrm{Ca} 2 \mathrm{p}$, Ti 2p, $\mathrm{O}$ 1s and $\mathrm{Na} 1 \mathrm{~s}$ edges. Figure 3 presents, TEM (Fig. 3a) and STXMbased $\mathrm{N}$ 1s imaging (Fig. 3b), along with $\mathrm{N}$ 1s spectra of selected regions (Fig. 3c). These reference spectra, when used to fit a full N 1s stack gave chemical maps displayed as a color composite in Fig 3d. The Ti nitride band, probably generated from a laser hardening surface treatment in air of the machined implant, is clearly visible, and correlated with the $\mathrm{N}$ signal in the APT measurement (Fig. 2c). Figure 4 presents results from STXM studies of the interface at the Ti $2 p$ and $\mathrm{Ca} 2 p$ edges. Both Ca $2 p$ EELS and STXM indicate the presence of multiple $\mathrm{Ca}$ species, with the spectrum of $\mathrm{Ca}$ at the interface being similar to that of amorphous calcium apatite (ACP) while that of $\mathrm{Ca}$ in the bone was similar to hydroxyapatite (HA). The Ti $2 \mathrm{p}$ signal was saturated in the bulk of the implant. However, two different Ti $2 p$ signals were observed in different regions of the interface, with one having a significantly larger 
$\mathrm{Ti}^{3+}$ character. Interestingly, a small $\mathrm{Ti} 2 \mathrm{p} \mathrm{TiO}_{\mathrm{x}}$ signal was observed throughout all of the bone region, potentially indicating that migration of $\mathrm{TiO}_{\mathrm{x}}$ into bone can occur over long time periods.

This paper has focused on a correlative study of a human bone - dental implant interface. Other recent correlative microscopy studies involving STXM include: TEM - STXM studies of greigite biomineralization [3], and an AFM - STXM - FTIR study of X-ray damage to perfluoro-sulfonate used as proton conducting ionomer in polymer-electrolyte membrane fuels cells. Together these results demonstrate the power of STXM as a complementary tool for correlative, multi-scale studies [4].

\section{References:}

[1] R. Brånemark, et al. J. Rehabil. Res. Dev. 38 (2001), p. 175.

[2] X. Wang et al. Advanced Materials Interfaces (2018) in review.

[3] X. Zhu et al. Geomicrobiology Journal 35 (2018), p. 215.

[4] ET, TEM-EELS and APT acquired at the Canadian Centre for Electron Microscopy (McMaster). STXM acquired at the Canadian Light Source, supported by CFI. Research funded by NSERC.

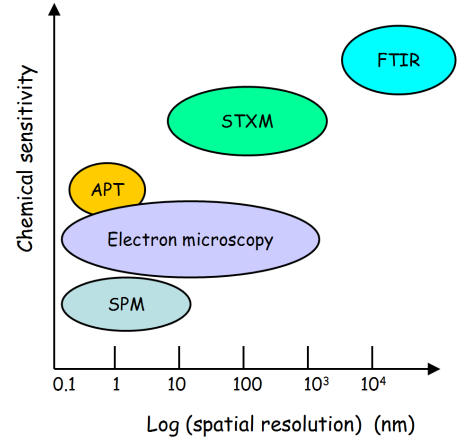

Figure 1. Spatial resolution and relative chemical sensitivity of correlative methods.

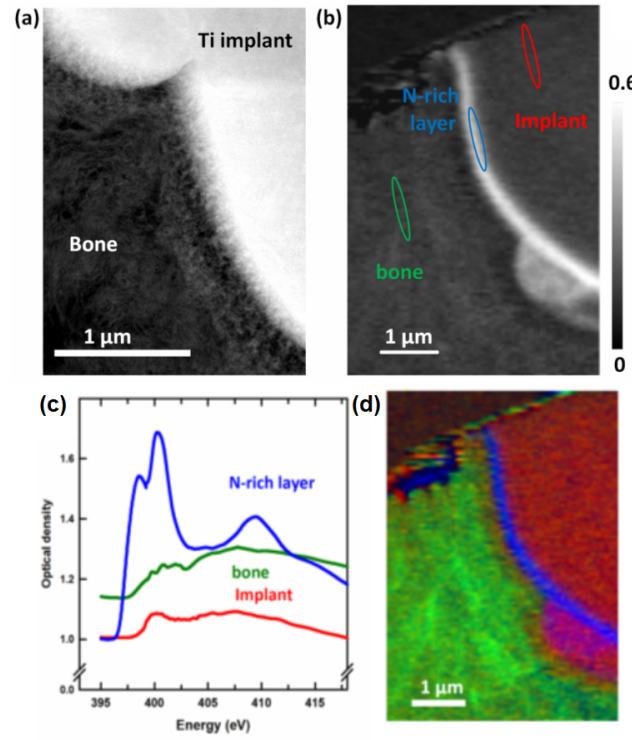

Figure 3. (a) STEM image, (b) STXM $\Delta$ image $\left(\mathrm{OD}_{400}-\mathrm{OD}_{396}\right),(\mathrm{c}) \mathrm{N}$ 1s spectra, (d) STXM chemical map based on N 1s spectra.

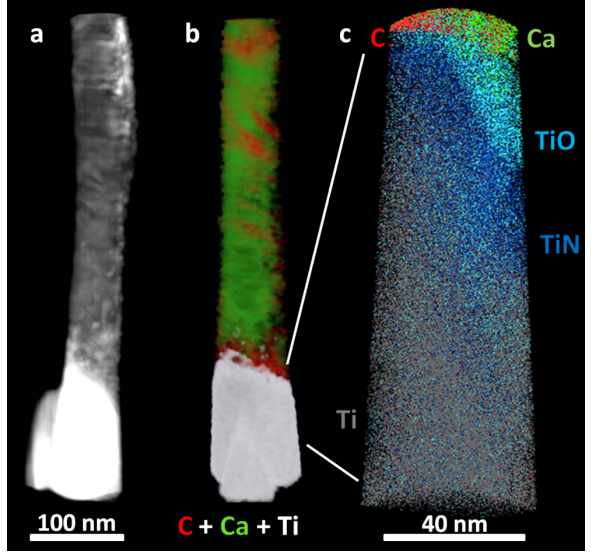

Figure 2. (a) On-axis ET, (b) EELS ET, (c) APT of the interface of human bone and a Ti dental implant.
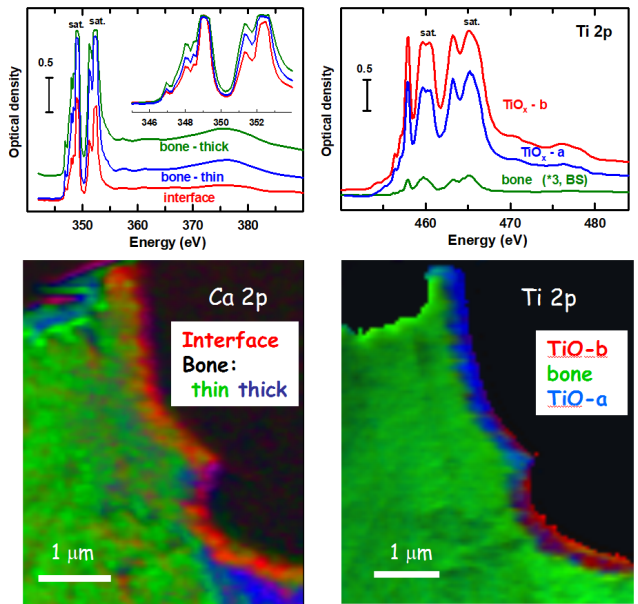

Figure 4. STXM speciation of bone-implant interface. (a) Ca $2 p$ spectra of interface. (b) maps of 3 Ca-containing components. (c) Ti $2 p$ spectra of interface. (d) maps of Ti components. 